\title{
A new version of targeted minicircle producer system for EBV-positive human nasopharyngeal carcinoma
}

\author{
YUFANG ZUO $^{1 *}$, SIHAI LIAO $^{1 *}$, ZUMIN XU $^{1 *}$, JIERONG XIE $^{1}$, WENLIN HUANG $^{2}$ and ZHONGHUA YU ${ }^{1}$ \\ ${ }^{1}$ Cancer Center, Affiliated Hospital of Guangdong Medical College, Zhanjiang, Guangdong; ${ }^{2}$ State Key Laboratory \\ of Oncology in South China, Cancer Center, Sun Yat-Sen University, Guangzhou, Guangdong, P.R. China
}

Received June 11, 2014; Accepted July 18, 2014

DOI: $10.3892 /$ or.2014.3486

\begin{abstract}
Targeted gene therapy needs to be implemented for future therapies to ensure efficient activity at the site of patient primary tumors or metastases without causing intolerable sideeffects. One of the elements of gene therapy is vector, which includes viral and non-viral vector. In the present study, we constructed a novel non-viral targeted gene therapeutic system by using the new minicircle (MC) producing plasmid for Epstein-Barr virus (EBV)-positive nasopharyngeal carcinoma (NPC). Molecular cloning technique was used to construct plasmids and electrophoretic analysis. Dual-luciferase reporter assay was used to evaluate the expression of luciferase. Fluorescence microscope was used to detect the expression of enhanced green fluorescence protein (EGFP). We constructed a new MC producing system pMC.BESPX-origin of plasmid replication (oriP), and demonstrated that this system could produce highly purified MC-oriP. Furthermore, our results showed that MC-oriP vector produced by the new system could mediate targeted luciferase gene expression in EBV-positive NPC cells. In addition, we verified that MC could mediate enhanced transgene expression compared with parent plasmid through EGFP transfection. The present study constructed a targeted expression vector pMC.BESPX-oriP which could
\end{abstract}

Correspondence to: Dr Zhonghua Yu, Cancer Center, Affiliated Hospital of Guangdong Medical College, Zhanjiang, Guangdong, P.R. China

E-mail: zhonghua_yu@126.com

Dr Wenlin Huang, State Key Laboratory of Oncology in South China, Cancer Center, Sun Yat-Sen University, Guangzhou, Guangdong, P.R. China

E-mail: hwenl@mail.sysu.edu.cn

${ }^{*}$ Contributed equally

Abbreviations: MC, minicircle; NPC, nasopharyngeal carcinoma; EGFP, enhanced green fluorescence protein; EBV, Epstein-Barr virus; EBNA1, Epstein-Barr nuclear antigen 1; oriP, origin of plasmid replication

Key words: targeted gene therapy, non-viral vector, minicircle, nasopharyngeal carcinoma, Epstein-Barr virus carry diversified therapeutic genes for EBV-positive NPC and provides a new approach for MC-based therapies.

\section{Introduction}

One of the most important objectives of gene therapy is the development of safe and efficient systems for gene transfer in eukaryotic cells. There are 2 strategies to provide target genes for gene transfer: viral-based and non-viral-based systems (1). Although viral-based systems have shown high transfection efficiency in vivo, they have serious disadvantages, such as immunogenicity and inflammatory response (2). Non-viral gene delivery strategies are usually based on plasmid DNA (pDNA) carrying the gene of interest. pDNA is an attractive platform for gene delivery since it is a non-viral, non-integrating vector that is safe, inexpensive, stable and easily manipulated (3). The popularity of pDNA continues to increase (18.3\% of trials compared to $18 \%$ in 2007 and $14 \%$ in 2004), and it is the most popular non-viral system used in clinical trials (4). Conventional plasmid vectors include a bacterial backbone and a transcription unit. However, these sequences may cause undesirable effects such as the production of antibodies against bacterial proteins expressed from cryptic upstream eukaryotic expression signals, changes in eukaryotic gene expression caused by the antibiotic resistance marker, and immune responses to $\mathrm{CpG}$ sequences $(5,6)$.

Compared to conventional plasmids, minicircle (MC) DNAs devoid of plasmid bacterial sequences are superior as non-viral DNA vector for multiple reasons: i) relative safety due to the reduced numbers of inflammatory unmethylated CpG motifs; ii) more efficient transgene expression due to its reduced size; and iii) more robust and persistent transgene expression (7-9). Previous studies have demonstrated that the use of MCs may offer a promising avenue for safe and efficacious non-viral-based gene therapies $(6,10-12)$.

The goal of cancer treatment is to selectively eliminate malignant cells while leaving normal tissues intact (13). Therefore, targeted strategies need to be implemented for future therapies to ensure efficient activity at the site of patient primary tumors or metastases without causing intolerable side-effects. Nasopharyngeal carcinoma (NPC) is prevalent in South China, North Africa and among Alaskan Eskimos. A unique feature of NPC is that nearly $100 \%$ of anaplastic or poorly differentiated NPCs contain Epstein-Barr virus (EBV) 
genomes and express EBV proteins (14), which are expressed exclusively in the malignant tissues but not in the surrounding normal tissues. This difference provides an exploitable opportunity for tumor-specific targeting. Initial genetic dissections of EBV identified one viral protein, Epstein-Barr nuclear antigen 1 (EBNA1), and one region of the viral genome, termed latent origin of plasmid replication (oriP), as being necessary and sufficient for replication of the viral plasmid. Previous studies have determined that EBNA1 is essential for regulating the transcription of the transforming genes of EBV (15-17). Additionally, EBNA-1, the only viral protein required for the replication of EBV in latently-infected cells, is found in all EBV-associated malignancies (18). The oriP is composed of two separable cis elements, the family of repeats (FR) and dyad symmetry element (DS) (19). The FR element consists of 20 tandem 30 -bp repeats and acts as a transcriptional enhancer for heterologous promoters when it is bound by EBNA1. Based on these features, the oriP-CMV promoter has been exploited for targeted gene therapy in EBV-positive NPC (20).

Due to these factors, we have developed a novel MC targeted therapy system in which transgene expression is under the transcriptional regulation of the oriP-CMV promoter (hereinafter referred to as oriP promoter). The binding of EBNA1 to the FR domain in oriP region activates the transcription of downstream genes. Selective expression of the therapeutic gene is successfully achieved in vitro and in vivo, indicating the feasibility of MC-oriP as a safe and highly effective gene therapy system for the treatment of NPC (21).

However, the major obstacle to widespread use of MCs is their time-consuming, labor-intensive production. In previous MC production schemes, the MC producer plasmid p2ФC31 contained a transgene expression cassette flanked with attB and attP, a set of inducible enzyme genes (a gene encoding homing endonuclease I-SceI and two copies of the gene encoding ФC31 integrase) and an I-SceI recognition site (9). The attB and attP sites are the bacterial and phage attachment sites of $\Phi С 31$ integrase, and the $\Phi С 31$ and I-SceI genes are regulated by the l-arabinose-inducible araCBAD system. MC DNA is generated by recombination between the attB and attP sites, and I-SceI initiates the destruction of the pDNA backbone circle by cutting through the engineered I-SceI site. Although the yields from this protocol were $\sim 1 \mathrm{mg}$ of $\mathrm{MC}$ DNA from 1 liter of overnight culture, the preparations still contained $\sim 3-15 \%$ of the input MC producer plasmid plus the plasmid backbone circle as contaminants. In addition to $\mathrm{CsCl}$ equilibrium gradient centrifugation to remove these unwanted DNAs, the production procedure is four labor-intensive days longer than routine plasmid production protocols (22).

Therefore, Chen et al presented a new system including the bacterial strain ZYCY10P3S2T plus the MC producer plasmid pMC.BESPX that allows simple, rapid and inexpensive production of a high-quality form of MCs. It enabled three improvements over the previous system (9). First, the procedure was simplified considerably and, compared to a routine plasmid preparation, required only an additional temperature change and 5-h incubation after addition of 1-arabinose. Second, the yield of MC was 3.4-4.8 mg/1,000 ml of overnight culture, making it $\sim 3-5$-fold higher than the previous MC producing system. In addition, compared with the previous
MC production protocol, there were 10 -fold fewer contaminating pDNAs, ranging from $0.4 \%$ to $1.5 \%$ of the input $\mathrm{MC}$ preparation. On a molar scale, the yield of MC was $20-70 \%$ higher than the MC producer plasmid. Third, the cost of MC production was similar to that of a standard plasmid. These production improvements, along with their superior expression profiles, make it feasible for MC DNA vectors to be used in place of pDNAs in mammalian expression studies (22).

In the present study, we developed a new targeted $\mathrm{MC}$ producing system by introducing targeted promoter oriP into the MC producer plasmid pMC.BESPX. Then, we demonstrated that this system could produce high-quality form of targeted MCs and the targeted MC containing oriP promoter could selectively express gene in vitro. To our knowledge, this is the first report on the new MC producer plasmid pMC. BESPX used in targeted gene expression. This system (pMC. BESPX-oriP) provides new targeted therapeutic vector for EBV-positive NPC.

\section{Materials and methods}

Construction of recombinant parent plasmids. Plasmid pMC. BESPX (4,084 bp) and bacterial strain ZYCY10P3S2T were gifts from Dr Zhiying Chen (Stanford University, Stanford, CA, USA) (22). Plasmid pSP72-oriP-luci-polyA was constructed by our laboratory (21). pcDNA3.1 (5,428 bp) and the E. coli strains top 10 were purchased from Invitrogen. pGL3-control was purchased from Promega. pEGFP-C2 (4.7 kb) was obtained from Clontech.

The 1-kb CMV-multiple cloning sites (MCS)-polyA fragment was amplified by PCR from pcDNA3.1 plasmid (Fig. 1A) and subcloned into the pMC.BESPX plasmid to create intermediate plasmid pMC.BESPX-CMV-MCS-polyA $(5.1 \mathrm{~kb}$, Fig. 1B). Then, parent plasmid pMC.BESPX-CMV-luci (6.7 kb, Fig. 1C) was constructed by replacing MCS of intermediate plasmid with luciferase gene $(1.7 \mathrm{~kb})$ obtained from plasmid pGL3-control (digested by HindIII and XbaI).

Parent plasmid pMC.BESPX-oriP-luci (6.9 kb) was constructed by inserting the $2.8-\mathrm{kb}$ SalI-oriP-luciferasepolyA-XhoI fragment from the intermediate plasmid pSP72-oriP-luci-polyA (Fig. 2A) into the SalI sites of pMC. BESPX; SalI and XhoI are isocaudamers (Fig. 2B).

Production and purification of MCs. MC-luciferase $(2.8-\mathrm{kb}$ MC-oriP-luci and 2.6-kb control MC-CMV-luci) were produced according to the methods described by Chen et al (22). Our present MC producing system, the strain ZYCY10P3S2T plus the MC producer plasmid pMC.BESPX-oriP-luci and pMC.BESPX-CMV-luci, allowed for a greatly simplified MC production protocol (Fig. 3A). On the morning of day 1, we inoculated cells from one transformed colony in $2 \mathrm{ml}$ of TB $(\mathrm{pH} 7.0)$ with $\mathrm{Kan}(50 \mu \mathrm{g} / \mathrm{ml})$ and incubated at $37^{\circ} \mathrm{C}$ with shaking at $250 \mathrm{rpm}$. Later that day, we amplified the bacteria by combining $25 \mu \mathrm{l}$ of culture to every $100 \mathrm{ml}$ TB containing Kan $(50 \mu \mathrm{g} / \mathrm{ml})$ and continued incubation overnight. At the end of the culture period, the OD600 was 4-5 with a $\mathrm{pH}$ of 6.5. On day 2, we prepared an $\mathrm{MC}$ induction mix comprising $100 \mathrm{ml}$ fresh LB, $4 \mathrm{ml} \mathrm{1N}$ sodium hydroxide and $0.1 \mathrm{ml} \mathrm{20 \%}$ L-arabinose and combined it with a $100 \mathrm{ml}$ overnight culture, and incubated the culture at $32^{\circ} \mathrm{C}$ with shaking at $250 \mathrm{rpm}$ for 
A

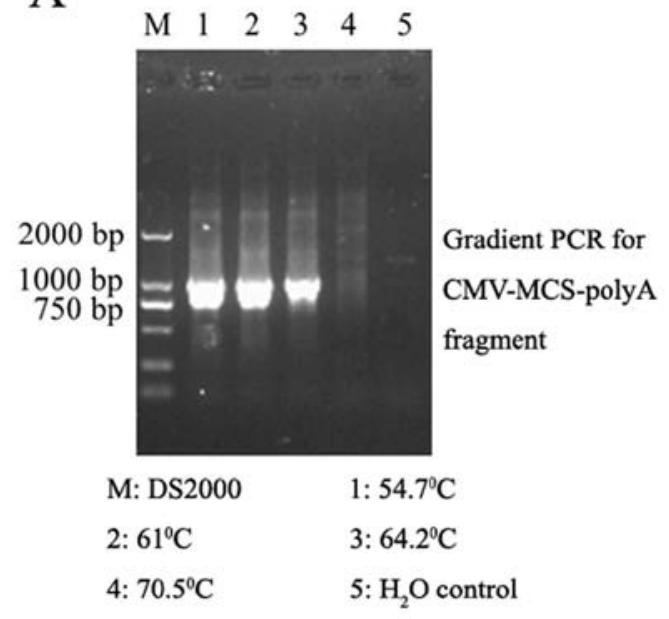

B

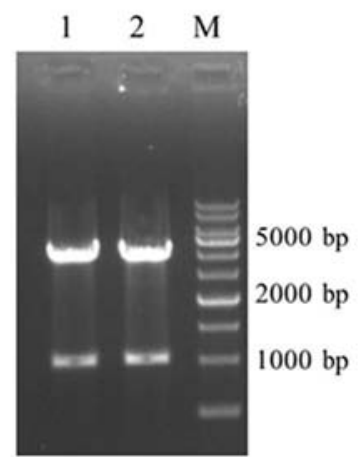

\author{
M: $1 \mathrm{~kb}$ ladder \\ 1: pMC.BESPX-CMV-MCS-polyA(clone1, BgIII+SalI) \\ 2: pMC.BESPX-CMV-MCS-polyA(clone11, BglII+SalI)
}

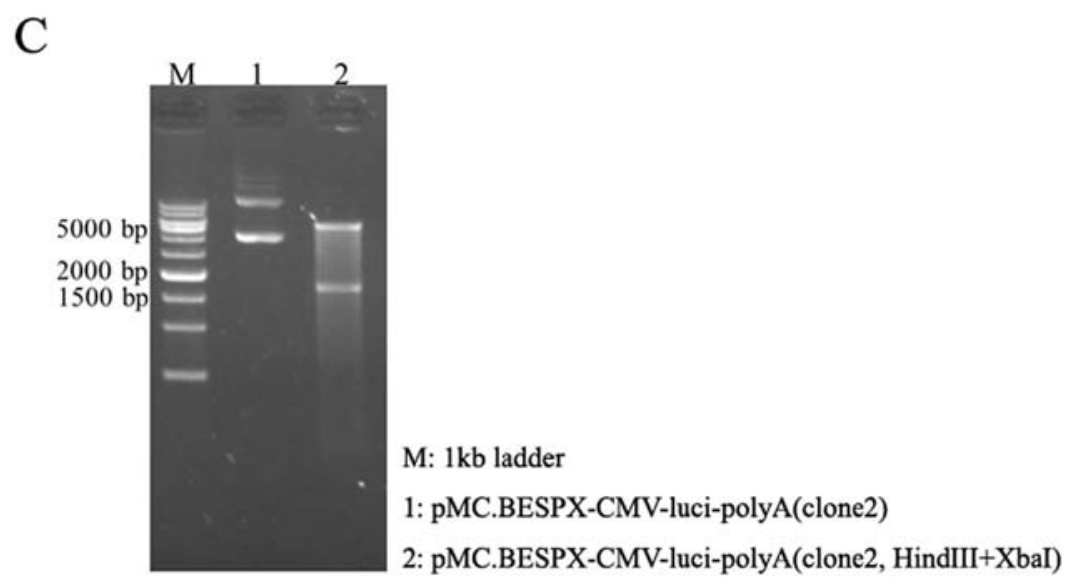

Figure 1. Construction of intermediate plasmid pMC.BESPX-CMV-MCS-polyA and control minicircle producer plasmid pMC.BESPX-CMV-luci-polyA. (A) Fragment CMV-MCS-polyA was amplified by PCR from pcDNA3.1 plasmid. PCR reactions were performed using the following primers: sense, 5'-GAAGATCTGTTGACATTGATTATTGACTAGTTATTAATAGTAAT-3' and antisense, 5'-GCGTCGACCCATAGAGCCCACCGCA TCCCCAGC-3'. The PCR reaction was carried out using the following conditions: denaturation at $94^{\circ} \mathrm{C}(40 \mathrm{sec})$, annealing at $64.5^{\circ} \mathrm{C}(30 \mathrm{sec})$ for extension at $72^{\circ} \mathrm{C}(60 \mathrm{sec})$ with 31 cycles. BglII (AGATCT) and SalI (GTCGAC) restriction enzymes were respectively added into the end of PCR fragment. The sizes of the PCR products were $\sim 1-k b$. (B) Restriction enzyme analysis of intermediate plasmid pMC.BESPX-CMV-MCS-polyA. Intermediate plasmid was created by inserting fragment CMV-MCS-polyA into empty parent plasmid pMC.BESPX with BglII and SalI restriction enzymes. Clone 1 and 11 are the positive plasmids of colony PCR (C) Restriction enzyme analysis of control minicircle producer plasmid pMC.BESPX-CMV-luci-polyA. Fragment luciferase gene was obtained from plasmid pGL3-control (digested by HindIII and XbaI). Then, plasmid pMC.BESPX-CMV-luci was constructed by replacing MCS of intermediate plasmid with luciferase gene. MCS, multiple cloning sites.

A

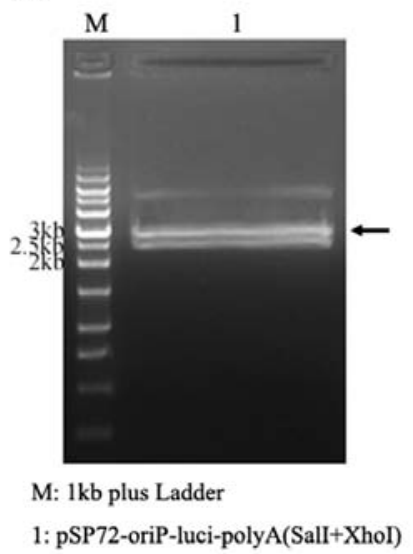

B

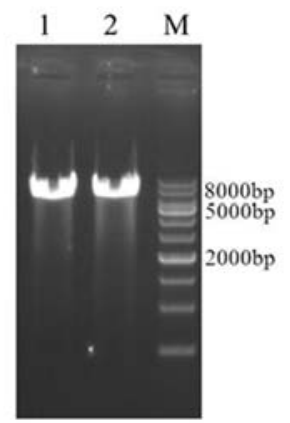

M: $1 \mathrm{~kb}$ ladder

1: pMC.BESPX-oriP-luci-polyA(clone 6, SalI)

2: pMC.BESPX-oriP-luci-polyA(clone 15 , Sall)

Figure 2. Construction of targeted minicircle producer plasmid pMC.BESPX-oriP-luci-polyA. (A) Fragment SalI-oriP-luciferase-polyA-XhoI was digested with SalI and XhoI from intermediate plasmid pSP72-oriP-luci-polyA which was constructed by our laboratory. (B) Restriction enzyme analysis of targeted minicircle producer plasmid pMC.BESPX-oriP-luci-polyA. It was constructed by inserting the SalI-oriP-luciferase-polyA-XhoI fragment into the SalI sites of pMC.BESPX (SalI and XhoI are isocaudamers). 

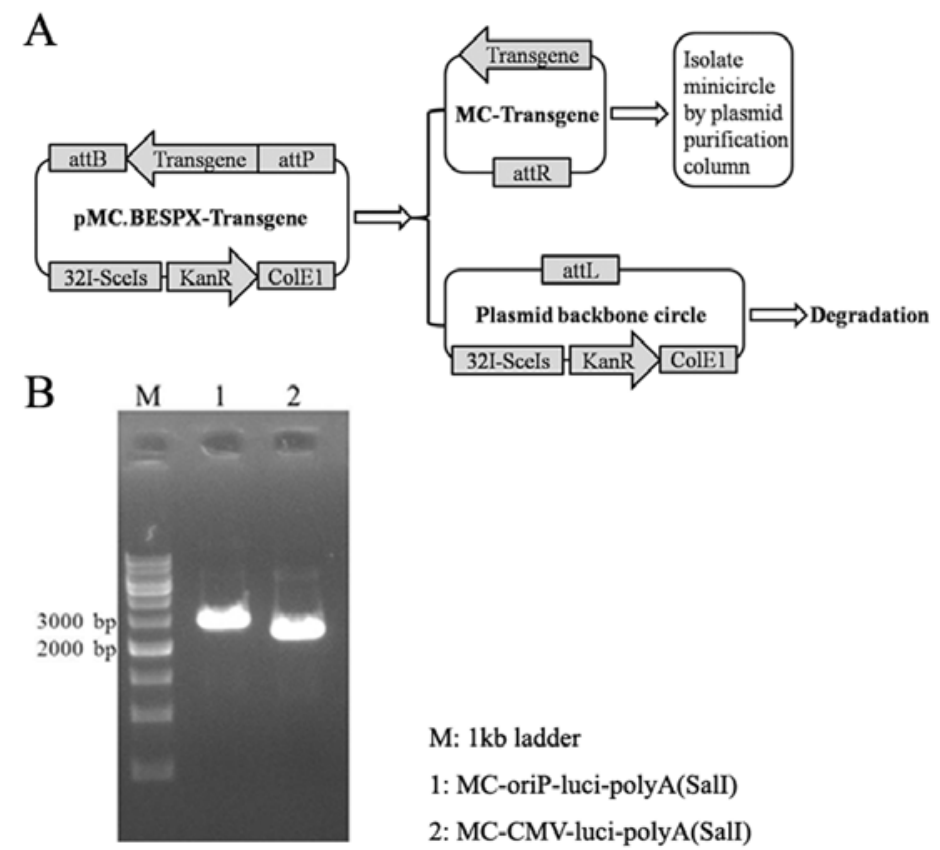

\author{
M: $1 \mathrm{~kb}$ ladder \\ 1: MC-oriP-luci-polyA(SalI) \\ 2: MC-CMV-luci-polyA(SalI)
}

Figure 3. Diagram of a minicircle production system and quality of the minicircle determined by gel analyses. (A) attB and attP, the bacterial and phage attachment sites of the $\Phi C 31$ integrase; attL, left hybrid sequence; attR, right hybrid sequence; I-SceI, I-SceI homing endonuclease gene; I-SceIs, the I-Scel recognition site; KanR, kanamycin resistance gene; ColE1, DNA replication origin. Minicircles were produced according to the protocol described in Materials and methods. (B) An agarose gel [0.8\% in Tris-acetate-EDTA (TAE) buffer] was made up to analyze the molecular weight and purity of minicircles digested by restriction enzyme SalI by comparing it with standard DNA marker (1 kb ladder, Dongsheng Biotech, China). One microliter of each sample that was diluted in $\mathrm{ddH}_{2} \mathrm{O}$ (DNA concentration $\sim 200 \mathrm{ng} / \mu \mathrm{l}^{-1}$ ) was added along with $5 \mu 1$ of $6 \mathrm{X}$ DNA loading dye to each well and subjected simultaneously to $110 \mathrm{mV}$ for up to $40 \mathrm{~min}$.

an additional $5 \mathrm{~h}$. We used Qiagen plasmid purification kits to isolate $\mathrm{MC}$ from bacterial lysates following the manufacturer's protocol (22).

Cells and culture conditions. The cell lines used in the present study were 293 (human embryonic kidney cell line, EBV-negative), NP69 (immortalized human nasopharyngeal epithelial cell line, EBV-negative), 5-8F (highly metastasized NPC cell line, EBV-negative), and C666-1 (undifferentiated and the only available EBV-positive NPC cell line) (23-25). 5-8F and C666-1 cells were maintained in RPMI-1640 containing $100 \mathrm{U} / \mathrm{ml}$ penicillin, $100 \mathrm{mg} / \mathrm{ml}$ streptomycin and $10 \%$ fetal bovine serum (Gibco, Paisley, Scotland) at $37^{\circ} \mathrm{C}$ in a $5 \% \mathrm{CO}_{2}$ humidified atmosphere. NP69 cells were maintained in Keratinocyte-SFM (cat. 10724; Gibco, Invitrogen), and the experiments were conducted when the cells were in an exponential growth phase. C666-1 was a gift from Dr Saiwah Tsao (University of Hong Kong, Hong Kong, China). NP69 and $5-8 \mathrm{~F}$ were kindly provided by Professor Musheng Zeng (State Key Laboratory of Oncology in South China, Cancer Center, Sun Yat-sen University, Guangzhou, China). The 293 cell line was maintained by our laboratory (21).

Quantitative assay of luciferase expression mediated by targeted or control promoter. To evaluate luciferase gene expression from MC-oriP-luci or MC-CMV-luci in EBV-negative and -positive cells, luciferase activity was measured using the Dual-Luciferase reporter assay system (Promega). Cells were seeded in 24-well culture plates the day before transfection. After one doubling, cells were cotransfected with different luciferase expressing plasmid and pGL4.73 (Promega) simultaneously at a ratio of 50:1. Cell lysates were analyzed for luciferase activity using the DualLuciferase reporter assay system and a Luminometer (Centro LB-960; Berthold Technologies) according to the manufacturers' protocols (21).

Fluorescence detection of egfp expression mediated by parent plasmid or MC plasmid. To further validate that MC mediated more efficient transgene expression compared with routine plasmid, we also constructed MC producer plasmid pMC.BESPX-CMV-EGFP, and isolated MC plasmid MC-CMV-EGFP. Transfections were conducted according to the manufacturer's instructions (Invitrogen). Twenty-four hours after transfection, fluorescence images of transfected 5-8F and C666-1 cells were captured using the Olympus IX71 motorized inverted microscope using FITC filter at X10 magnification (26).

Statistical analysis. All results were evaluated using Student's t-test with SPSS 17.0 software (SSPS, Inc., Chicago, IL, USA). $\mathrm{p}<0.05$ was considered to indicate a statistically significant difference. Representative results from three independent experiments are shown, and the data are presented as means $\pm \mathrm{SD}$.

\section{Results}

Analysis of molecular weight and purity of MCs by gel electrophoresis. MC DNA produced by using designated protocols was purified using Qiagen plasmid purification kits and analyzed by gel electrophoresis (Fig. 3B). Prior to electropho- 
A

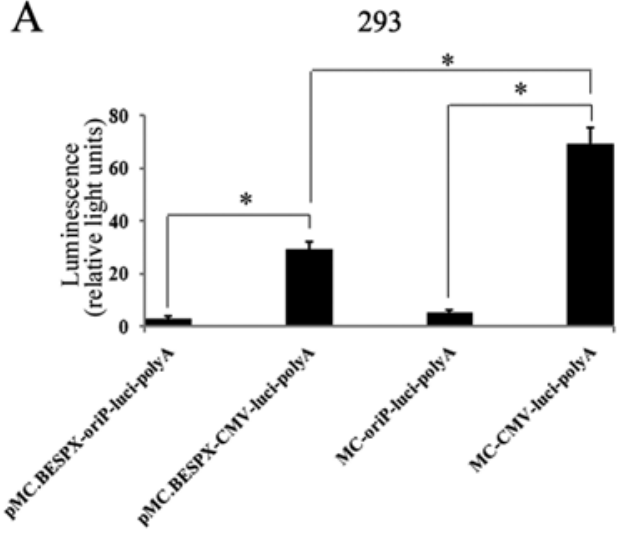

$\mathrm{C}$

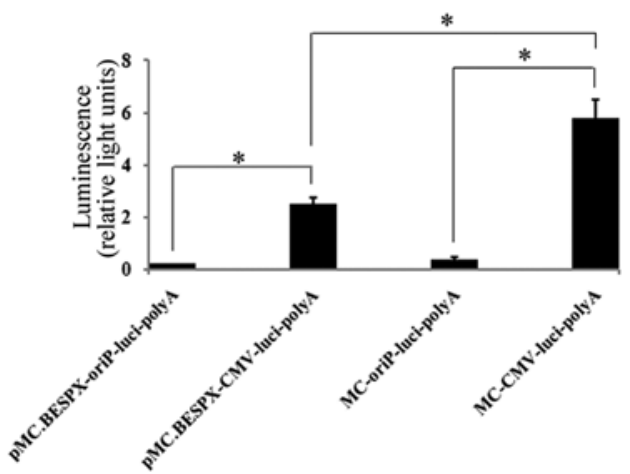

B

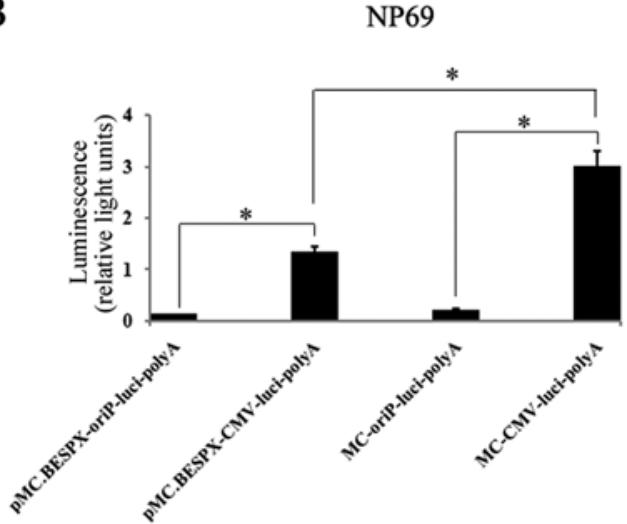

$\mathrm{D}$

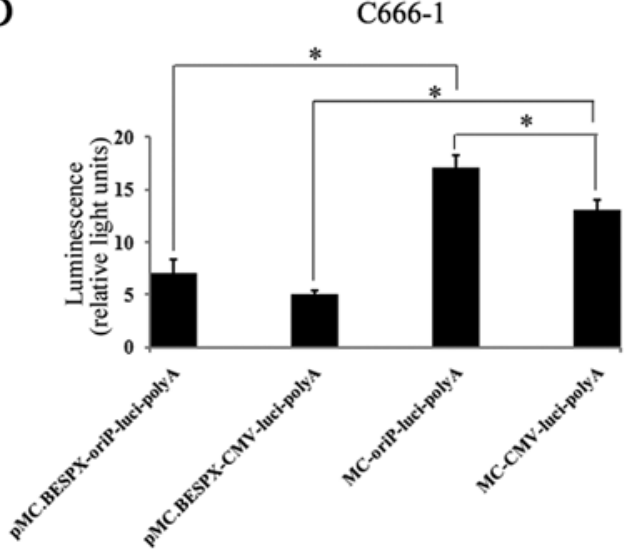

Figure 4. Selective expression of minicircle-oriP vector produced by novel producing system. (A, B and C) Luciferase activities were 10 to 20 -fold lower when driven by oriP promoter than by CMV promoter in EBV-negative cells $(\mathrm{p}<0.001)$. (D) In contrast, luciferase activity was higher when driven by oriP promoter than by CMV promoter in the EBV-positive C666-1 cells $(\mathrm{p}<0.05)$.

resis, DNAs were digested with restriction enzyme SalI. As shown in Fig. 3, the size of MCs was close to their expression cassette (oriP-luciferase-polyA and CMV-luciferase-polyA). Notably, there was no contaminating pDNA by macroscopic observation. This is consistent with a previous report (22).

Selective expression of luciferase in EBV-positive C666-1 cells mediated by oriP-vectors. To determine the transgene expression provided by the novel EBNA1-regulated MC vectors in EBV-negative cells (293, NP69 and 5-8F cells), and the only available EBV-positive NPC cell line (C666-1), the MC-luci was compared with its parent plasmid pMC.BESPX-luci. All these plasmids contained a luciferase expression cassette driven by an oriP promoter or cytomegalovirus promoter (Fig. 4). Luciferase activity was assessed $48 \mathrm{~h}$ (72 h for C666-1 due to its low growth rate) after transfection using the DualLuciferase reporter assay system. Luciferase activities were 10-20-fold lower when driven by oriP promoter than by CMV promoter in EBV-negative cells ( $<<0.001$; Fig. 4). In contrast, luciferase activity was higher when driven by oriP promoter than by CMV promoter in the EBV-positive C666-1 cells (p<0.05; Fig. 4). Occasionally, low levels of luciferase activity were detected in some EBV-negative cells when driven by the oriP promoter, which suggested the basal expression induced by the minimal CMV IE promoter included in oriP promoter. This observation is consistent with a previous study (20). The expression levels of the MC groups were significantly higher

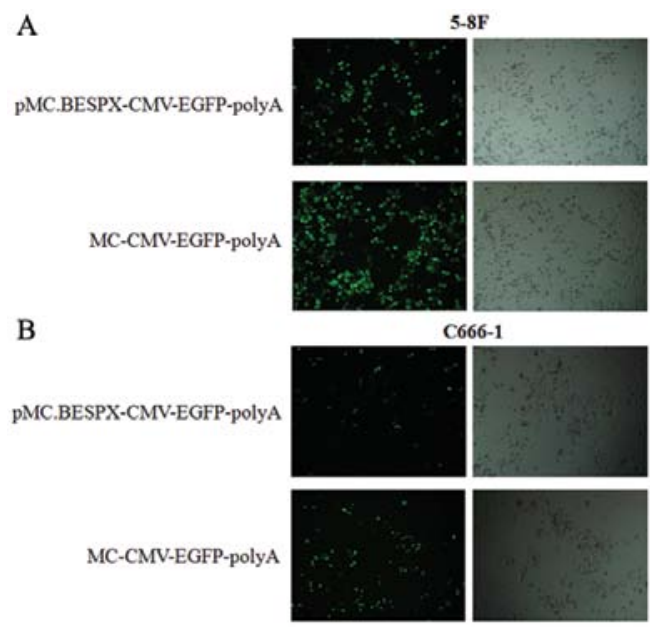

Figure 5. Enhanced transfected efficiency of minicircle compared with parent groups in vitro. Parent plasmid pMC.BESPX-CMV-EGFP $(5.8 \mathrm{~kb})$ was constructed by replacing MCS of intermediate plasmid pMC.BESPX-CMVMCS-polyA with egfp gene ( $760 \mathrm{bp})$ obtained from plasmid pEGFP-C2 (digested by NheI + HindIII). MC-CMV-EGFP was produced according to the protocol described in Materials and methods. Fluorescent microscopy images of transfected 5-8F and C666-1 cells with minicircle producer plasmid and minicircle DNA after $24 \mathrm{~h}$.

than those of the corresponding parent groups, demonstrating that the $\mathrm{MC}$ is more efficient in mediating transgene expression in vitro $(\mathrm{p}<0.01 ;$ Fig. 4$)$. 
Enhanced expression of MC-encoded egfp gene compared with parent plasmid in vitro. The transfection efficiency of parent plasmid and $\mathrm{MC}$ were compared in $5-8 \mathrm{~F}$ (Fig. 5A) and C666-1 (Fig. 5B) cells using Lipofectamine 2000 (Invitrogen). Twenty-four hours after transfection, there was a significant increase in GFP expression in the MC-transfected cell population compared with the parent plasmid-transfected cell population (Fig. 5).

\section{Discussion}

Our data indicate the novel minicircle (MC) producing system pMC.BESPX-oriP could produce high-quality form of MC-oriP. Targeted and increased gene expression is successfully achieved in MC-oriP-transfected EBV-positive cells. More efficient transgene expression is reconfirmed in MC-EGFP compared with pMC.BESPX-EGFP. The novelty of the present study lies in the introduction of oriP promoter into the new MC producing system pMC.BESPX and application of this system in gene expression in EBV-positive nasopharyngeal carcinoma (NPC).

Gene therapy is an emerging field in medical and pharmaceutical sciences due to its potential to treat chronic diseases such as cancer, viral infections, myocardial infarctions and genetic disorders. Application of gene therapy is limited due to lack of suitable methods for proper introduction of genes into cells and, therefore, this is an area of interest for most researchers. To achieve successful gene therapy, development of proper gene delivery systems may be one of the most important factors. Several non-viral and viral gene transfer methods have been developed. Although the viral agents have a high transferring efficiency, they are difficult to handle due to their toxicity. To overcome the safety problems of the viral counterpart, several non-viral in vitro and in vivo gene delivery systems have been developed (27). Plasmid DNA (pDNA) is the most popular non-viral system used in clinical trials (4).

Standard pDNA has two components: the bacterial backbone required for plasmid propagation in bacteria and the transcription cassette for expression in mammalian cells. An MC episomal DNA vector is a circular expression cassette devoid of the bacterial pDNA backbone. pDNA size is inversely related to transfection efficiency and correspondingly, the transfection efficiency and expression levels of MC are higher than pDNA levels (12). MCs have been used for years in preclinical gene transfer research due to their 10-1,000-fold enhancement compared with regular plasmids in long-term transgene expression in quiescent tissues in vivo and in vitro (22). Owing to this advantage, Huang et al created MC carrying HIF-1 $\alpha$ (MC-HIF-1 $\alpha$ ) therapeutic gene for treatment of myocardial infarction. They also demonstrated that MC could significantly improve transfection efficiency, duration of transgene expression and cardiac contractility (6). Jia et al used a single MC vector to generate transgene-free induced pluripotent stem cells (iPSCs) from adult human adipose stem cells (10). Gao et al developed a magnetic resonance imaging (MRI) visible nanoparticle to monitor MC DNA gene delivery and explore the potential of gene therapy in vivo (28). Dietz et al also demonstrated that MC DNA is superior to pDNA in eliciting antigen-specific $\mathrm{CD} 8^{+} \mathrm{T}$-cell responses (12).
In our previous study, we generated a safe and highly effective gene therapy system for NPC by using the MC producer plasmid p2 $\Phi C 31$, which was provided by Dr Zhiying Chen (Stanford University) (21). The MC preparations contained $\sim 3-15 \%$ of the input MC producer plasmid plus the plasmid backbone circle as contaminants. A new system pMC.BESPX and the bacterial strain ZYCY10P3S2T that allow simple, rapid and inexpensive production of a high-quality form of MCs has been presented (22).

In the present study, we constructed a targeted MC producing system by using the novel parent plasmid pMC.BESPX and the bacterial strain ZYCY10P3S2T. We isolated MCs without macroscopic plasmid contamination (Fig. 3B). Our results verified the superiority of pMC.BESPX in MC preparation, and is consistent with other reports $(12,22)$. Then, we detected the targeted gene expression of pMC.BESPX-oriP and MC-oriP in EBV-negative and EBV-positive cells. Both in parent plasmid and in MC group, oriP promoter could mediate selective gene expression compared to CMV promoter (Fig. 4). This indicates that $\mathrm{MC}$-oriP which is produced by the new producing system constructed in the present study, could also mediate targeted gene expression. It is similar to our previous study (21). Finally, we verified that $\mathrm{MC}$ was more efficient in gene expression compared with parent plasmid (Fig. 5). Our results suggest that pMC.BESPX-oriP could carry various therapeutic gene and produce high-quality $\mathrm{MC}$ which can be used in targeted gene therapy for EBV-positive NPC.

In conclusion, we showed for the first time the application of targeted promoter oriP in the novel $\mathrm{MC}$ producing plasmid pMC.BESPX, and demonstrated the selective expression of pMC.BESPX-oriP, as well as its inducing product MC-oriP. We constructed a targeted expression vector which could carry diversified therapeutic gene for EBV-positive NPC. The present study provides a new approach toward MC-based therapies.

\section{Acknowledgements}

The authors thank Dr Zhiying Chen (Stanford University, Stanford, CA, USA) for his generous gift of plasmid pMC. BESPX and bacterial strain ZYCY10P3S2T. This study was supported by grants from the Open Project Program of State Key Laboratory of Oncology in Southern China (HN2012-06), the PhD Programs Foundation of Guangdong Medical College (XB1228), and the National Natural Science Foundation of China (no. 81201736).

\section{References}

1. Lyon AR, Sato M, Hajjar RJ, Samulski RJ and Harding SE: Gene therapy: targeting the myocardium. Heart 94: 89-99, 2008.

2. Marshall E: Gene therapy death prompts review of adenovirus vector. Science 286: 2244-2245, 1999.

3. Faurez F, Dory D, Le Moigne V, Gravier R and Jestin A: Biosafety of DNA vaccines: new generation of DNA vectors and current knowledge on the fate of plasmids after injection. Vaccine 28: 3888-3895, 2010.

4. Ginn SL, Alexander IE, Edelstein ML, Abedi MR and Wixon J: Gene therapy clinical trials worldwide to 2012 - an update. J Gene Med 15: 65-77, 2013.

5. Jechlinger W: Optimization and delivery of plasmid DNA for vaccination. Expert Rev Vaccines 5: 803-825, 2006.

6. Huang M, Chen Z, Hu S, et al: Novel minicircle vector for gene therapy in murine myocardial infarction. Circulation 120 (Suppl 11): S230-S237, 2009. 
7. Chen ZY, He CY, Ehrhardt A and Kay MA: Minicircle DNA vectors devoid of bacterial DNA result in persistent and highlevel transgene expression in vivo. Mol Ther 8: 495-500, 2003.

8. Chen ZY, He CY, Meuse L and Kay MA: Silencing of episomal transgene expression by plasmid bacterial DNA elements in vivo. Gene Ther 11: 856-864, 2004.

9. Chen ZY, He CY and Kay MA: Improved production and purification of minicircle DNA vector free of plasmid bacterial sequences and capable of persistent transgene expression in vivo. Hum Gene Ther 16: 126-131, 2005.

10. Jia F, Wilson KD, Sun N, et al: A nonviral minicircle vector for deriving human iPS cells. Nat Methods 7: 197-199, 2010.

11. Zhang C, Gao S, Jiang W, et al: Targeted minicircle DNA delivery using folate-poly(ethylene glycol)-polyethylenimine as non-viral carrier. Biomaterials 31: 6075-6086, 2010

12. Dietz WM, Skinner NE, Hamilton SE, et al: Minicircle DNA is superior to plasmid DNA in eliciting antigen-specific CD8 T-cell responses. Mol Ther 21: 1526-1535, 2013.

13. Hine CM, Seluanov A and Gorbunova V: Use of the Rad51 promoter for targeted anti-cancer therapy. Proc Natl Acad Sci USA 105: 20810-20815, 2008

14. Cohen JI: Epstein-Barr virus infection. N Engl J Med 343: 481-492, 2000

15. Bochkarev A, Barwell JA, Pfuetzner RA, Bochkareva E, Frappier L and Edwards AM: Crystal structure of the DNA-binding domain of the Epstein-Barr virus origin-binding protein, EBNA1, bound to DNA. Cell 84: 791-800, 1996.

16. Niedobitek G, Agathanggelou A and Nicholls JM: EpsteinBarr virus infection and the pathogenesis of nasopharyngea carcinoma: viral gene expression, tumour cell phenotype, and the role of the lymphoid stroma. Semin Cancer Biol 7: 165-174, 1996.

17. Altmann M, Pich D, Ruiss R, Wang J, Sugden B and Hammerschmidt W: Transcriptional activation by EBV nuclear antigen 1 is essential for the expression of EBV's transforming genes. Proc Natl Acad Sci USA 103: 14188-14193, 2006.
18. Leight ER and Sugden B: EBNA-1: a protein pivotal to latent infection by Epstein-Barr virus. Rev Med Virol 10: 83-100, 2000.

19. Lindner SE and Sugden B: The plasmid replicon of Epstein-Barr virus: mechanistic insights into efficient, licensed, extrachromosomal replication in human cells. Plasmid 58: 1-12, 2007.

20. Li JH, Chia M, Shi W, et al: Tumor-targeted gene therapy for nasopharyngeal carcinoma. Cancer Res 62: 171-178, 2002.

21. Zuo Y, Wu J, Xu Z, et al: Minicircle-oriP-IFN $\gamma$ : a novel targeted gene therapeutic system for EBV positive human nasopharyngeal carcinoma. PLoS One 6: e19407, 2011.

22. Kay MA, He CY and Chen ZY: A robust system for production of minicircle DNA vectors. Nat Biotechnol 28: 1287-1289, 2010.

23. Teng ZP, Ooka T, Huang DP and Zeng Y: Detection of EpsteinBarr virus DNA in well and poorly differentiated nasopharyngeal carcinoma cell lines. Virus Genes 13: 53-60, 1996.

24. Liu T, Ding Y, Xie W, et al: An imageable metastatic treatment model of nasopharyngeal carcinoma. Clin Cancer Res 13: 3960-3967, 2007.

25. Cheung ST, Huang DP, Hui AB, et al: Nasopharyngeal carcinoma cell line (C666-1) consistently harbouring Epstein-Barr virus. Int J Cancer 83: 121-126, 1999.

26. Dong Y, Aied A, Li J, Wang Q, Hu X and Wang W: An in vitro approach for production of non-scar minicircle DNA vectors. J Biotechnol 166: 84-87, 2013.

27. Manjila SB, Baby JN, Bijin EN, Constantine I, Pramod K and Valsalakumari J: Novel gene delivery systems. Int J Pharm Investig 3: 1-7, 2013

28. Gao L, Xie L, Long X, et al: Efficacy of MRI visible iron oxide nanoparticles in delivering minicircle DNA into liver via intrabiliary infusion. Biomaterials 34: 3688-3696, 2013. 